\title{
Association Between Surrogate Index of Insulin Resistance and Coronary Collateral Circulation in Patients with Coronary Chronic Total Occlusion Lesions
}

Jinxing Liu

Beijing An Zhen Hospital: Capital Medical University Affiliated Anzhen Hospital

Chengping $\mathrm{Hu}$

Beijing An Zhen Hospital: Capital Medical University Affiliated Anzhen Hospital

Yan Liu

Beijing An Zhen Hospital: Capital Medical University Affiliated Anzhen Hospital

Yong Zhu

Beijing An Zhen Hospital: Capital Medical University Affiliated Anzhen Hospital

Hongya Han

Beijing An Zhen Hospital: Capital Medical University Affiliated Anzhen Hospital

Yujie Zhou

Beijing An Zhen Hospital: Capital Medical University Affiliated Anzhen Hospital

Yingxin Zhao ( $\nabla$ zyingxinmi@163.com )

Beijing An Zhen Hospital: Capital Medical University Affiliated Anzhen Hospital

\section{Research Article}

Keywords: Triglyceride glucose index, Insulin resistance, Chronic total occlusion, coronary collateralization circulation

Posted Date: September 2nd, 2021

DOl: https://doi.org/10.21203/rs.3.rs-853157/v1

License: (c) (1) This work is licensed under a Creative Commons Attribution 4.0 International License. Read Full License 


\section{Abstract}

Background: Recent studies have substantiated the role of triglyceride glucose index (TyG index) in the prognosis of coronary artery disease (CAD) patients while no relevant studies revealed the association between TyG index and coronary collateral circulation (CCC) in the event of coronary chronic total occlusion (CTO). Our study intends to explore whether or to what extent TyG index is associated with less developed CCC in CAD patients with CTO lesions.

Methods: The study enrolled 1093 ACS patients undergoing percutaneous coronary intervention (PCI) for at least one CTO lesion. Relevant data was collected from Beijing Anzhen Hospital record system. The degree of collaterals was determined according to Rentrop classification system. The correlation between TyG index and coronary collateralization was assessed.

Results: Overall, 318 patients were divided into poor CCC group. TyG index was significantly higher in patients with poor $\operatorname{CCC}(9.3 \pm 0.65$ vs. $8.8 \pm 0.53, \mathrm{P} \otimes 0.001)$. After adjusting for various confounding factors, TyG index remained to be correlated with the occurrence of poor CCC, with an ORs (95\% Cls) of 1.59 (1.07-2.36) in T2 group and 5.72 (3.83-8.54) in T3 group compared with the first tertile. Besides, subgroup analysis showed that higher TyG index values remained to be strongly associated with increased risks of poor CCC. Lastly, to compare the risk assessment efficacy for the formation of CCC between TyG index and glycosylated hemoglobin $\mathrm{A} 1 \mathrm{c}(\mathrm{HbA} 1 \mathrm{c})$ and another insulin resistance (IR) surrogate marker triglyceride-to-high-density lipoprotein cholesterol ratio, area under the receiver-operating characteristic (ROC) curve (AUC) was obtained, a significant improvement on the risk assessment performance for the poor CCC emerged when adding TyG index into a baseline model [AUC, 0.629 for baseline model vs. 0.724 for baseline model + TyG index vs. 0.638 for baseline model +HbA1c vs. 0.721 for baseline model $+\mathrm{TG} / \mathrm{HDL}-\mathrm{C}, \mathrm{P}<0.001$; net reclassification improvement (NRI) for TyG index, 0.238, P₫0.001; integrated discrimination improvement (IDI) for TyG index, 0.103, P®0.001].

Conclusions: The TyG index is strongly associated with the occurrence of poor CCC in CAD patients with CTO lesions and its risk assessment performance is better than $\mathrm{HbA} 1 \mathrm{c}$ and TG-to-HDL-C ratio.

\section{Background}

Coronary artery disease (CAD), refers to the accumulative process of atherosclerotic plaques in the epicardial arteries, is a major cause of death worldwide. ACS, usually due to the abrupt plaque rupture or erosion, is a serious clinical presentation of CAD and needs to take active management strategies. In recent years, the early application of invasive strategies, such as $\mathrm{PCl}$, coronary artery bypass grafting (CABG) and antithrombotic treatments, has greatly decreased the in-hospital major adverse cardiovascular events (MACE) and mortality rates[1]. However, 5\%-10\% patients with CAD continue to experience the symptom of angina pectoris despite the optimal drug therapy and revascularization strategies, namely "no-option" patients[2]. Reasons for patients not suitable for revascularization includes advanced age, the presence of multiple comorbidities and most importantly, complicated coronary lesions such as CTO. According to the report from the National Cardiovascular Data Registry (NCDR)[3], 
the successful rate of recanalization of CTO lesions is significantly lower than non-CTO patients (55\% vs. $96 \%, \mathrm{P}<0.001$ ) and patients undergoing CTO PCI requires higher contrast volume and longer fluoroscopy time, which exerts unfavorable effect on short- and long-term prognosis of patients, especially for those aged and with chronic kidney disease (CKD). Besides, CTO lesion is associated with the increased risk of In-Stent Restenosis (ISR)[4]. Hence, invasive revascularization therapy may not be the optimal option for those patients. Coronary collateralization, as a network of arterial-arterial anastomotic connection between major epicardial arteries, could undergo abluminal expanding and wall thickening to expand its diameter to a larger caliber in the presence of severe obstructive CAD, which could effectively alleviate the symptoms caused by cardiac ischemia[5]. Studies have shown the protective role of good CCC in improving the survival and prognosis in patients with CAD, such as improved long-term survival, lower cardiac death and MACE rates[6, 7]. While the formation of CCC is easily affected by many factors, people with different clinical characteristics have a tendency to develop impaired coronary collateral growth in prominent epicardial arteries stenosis[8, 9]. Previous study has substantiated there is an inverse association between coronary collateral growth and the presence of diabetes, components of metabolic syndrome (MetS), or its key parameter IR[10]. HOMA-IR, an indicator of IR, which is calculated as fasting glucose $\times$ fasting insulin / 22.5, is not very applicable for routine clinical practice because fasting insulin is not a common laboratory measurement for CAD patients, especially for those with non-diabetes. Besides, no relevant studies have explored the association between IR and CCC in CAD patients with CTO lesions. Hence, identifying a simple surrogate index of IR to evaluate the formation CCC is of great clinical significance.

TyG index, whose value is mainly determined by the fasting triglyceride and glucose levels, has been recognized as a novel surrogate marker of IR and had been proved to be strongly associated with traditional IR markers, such as HOMA-IR[11]. Evidence showed that TyG index can predict the presence of diabetes, prediabetes and MetS[12,13]. Recent studies has focused on the association between TyG index and cardiovascular complications and outcomes in those with CAD and shows a significant correlation between TyG index and coronary artery calcification[14], arterial stiffness[15] and long-term cardiovascular events in patients with $\mathrm{CAD}[16-19]$. However, to date there is no exclusive study focusing on the relationship between TyG index and coronary collateral growth in those CAD patients with CTO lesions. Hence, this study aims to investigate the association between TyG index and coronary collateral grading and compare its risk diagnostic value with that of type 2 mellitus diabetes (T2DM) marker HbA1c and another IR surrogate index TG-to-HDL-C ratio.

\section{Methods}

\section{Study population}

A total of 1513 patients who were diagnosed as CAD and with CTO ( $>3$ months) of at least one major epicardial coronary artery at Beijing AnZhen Hospital between 1st Jan 2020 and 31st Dec 2020 were recruited into this single-center, observational study (As is shown in Fig. 1). Coronary CTO lesion was defined as a coronary lesion with Thrombolysis in Myocardial Infarction (TIMI) flow grade 0 of at least 3 
months[20]. Estimation of the duration of occlusion was based on the occurrence of myocardial infarction (MI) in the territory supplied by the occluded vessels, an abrupt worsening of existing angina or the comparison with the prior angiogram. For the purpose of this study, patients were excluded because of a history of CABG $(n=283)$, acute infectious disease $(n=3)$, tumor or immune system disease $(n=25)$, suspected familial hypertriglyceridemia $(n=20)$, estimated glomerular filtration rate $($ eGFR) $<30$ $\mathrm{mL} /\left(\operatorname{minX} 1.73 \mathrm{~m}^{2}\right)$ or severe hepatic insufficiency $(n=11)$, Type 1 Diabetes Mellitus $(n=1)$, and left main coronary artery disease or NYHA III or IV or LVEF $<30 \%(n=16)$; Besides, 61 patients were excluded for the reasons of lacking some basic laboratory or demographic data; Overall, 1093 participants were finally enrolled for the present analysis. The detailed enrolled procedure was depicted in Fig. 1.

\section{Disease definition}

The diagnosis of Type 2 Diabetes Mellitus (T2DM) is based on the previous diagnosis and treatment with glucose-lowering medication or recommendations from the American Diabetes Association (ADA), which includes 1) fasting plasma glucose (FPG) $\geq 126 \mathrm{mg} / \mathrm{dL}(7.0 \mathrm{mmol} / \mathrm{L}) ; 2) 2 \mathrm{~h}-\mathrm{PG} \geq 200 \mathrm{mg} / \mathrm{dL}$ (11.1 mmol/L) during OGTT (75g oral glucose tolerance test); 3 ) HbA1c $\geq 6.5 \%$; 4 ) classic symptom of hyperglycemia or hyperglycemic crisis, a random plasma glucose $\geq 200 \mathrm{mg} / \mathrm{dL}(11.1 \mathrm{mmol} / \mathrm{L})[21]$. Hypertension was defined by the recommendations from the Europe Society of Cardiology (ESC) / European Society of Hypertension (ESH): office systolic blood pressure (SBP) value $\geq 140 \mathrm{mmHg}$ and / or diastolic blood pressure (DBP) values $\geq 90 \mathrm{mmHg}$ or the use of antihypertensive agents in the past 2 weeks[22]. Dyslipidemia was characterized by increased total cholesterol (TC) or low-density lipoprotein cholesterol (LDL-C) and triglycerides (TG), or decreased high-density lipoprotein cholesterol (HDL-C) according to the third report of the National Cholesterol Education Program (NCEP)[23]. MetS is defined according to the guideline for T2DM by Diabetes Branch of the Chinese Medical Association[24], using body mass index (BMI) for the replacement of waist circumference or obesity, including three or more of the following abnormalities: 1 ) high blood pressure $\geq 130 / 85 \mathrm{mmHg}$ or treatment with antihypertensive drugs); 2) hypertriglyceridemia ( serum triglyceride $\geq 1.70 \mathrm{mmol} / \mathrm{L}$ or $150 \mathrm{mg} / \mathrm{dL}$ ); 3) low HDL-C $<1.04$ $\mathrm{mmol} / \mathrm{L} ; 4)$ elevated fasting glucose ( $\geq 6.1 \mathrm{mmol} / \mathrm{L}$ ) or treated with antidiabetic drugs; 5$)$ overweight or obesity $\left(\mathrm{BMI} \geq 25 \mathrm{Kg} / \mathrm{m}^{2}\right)$

\section{Demographic and biochemical measurements}

Demographic data like sex, age, BMI, SBP, DBP, smoking, hypertension, T2DM, hypertriglyceridemia, hypercholesterolemia, previous history of $\mathrm{MI}$ or stroke and medication history was collected from Beijing Anzhen hospital medical information record system; left ventricular ejection fraction (LVEF) is calculated by echocardiography at admission; Blood samples were taken after overnight fasting ( $>\mathrm{h}$ ). Serum levels of lipid profiles, fasting plasma glucose (FPG), glycosylated albumin (GA), HbA1c, uric acid (UA) and highsensitivity C-reactive protein (hs-CRP) were all calculated by standard laboratory techniques; The enzymatic hexokinase method was used to calculate FPG; Lipid profiles like TC, TG, HDL-C and LDL-C were measured by standard enzymatic methods. The non-high-density lipoprotein cholesterol (non-HDLC) was calculated as TC minus HDL-C. The TyG index was calculated as the In (FPG level [mg/dL] 
xfasting TG levels [mg/dL] / 2). TG-to-HDL-C ratio was calculated as TG/HDL-C. The study protocol was approved by the Institutional Review Board of The Anzhen Hospital, Beijing, China.

\section{Angiography and collateral classification}

The formation of CCC in CAD patients with CTO lesions was determined by coronary angiography (CAG), which was performed via the radial or femoral access using 6F diagnostic catheters. The results of angiogram were independently evaluated and explained by two experienced interventional cardiologists who were blinded for this study. Rentrop scoring system was used to assess the grading of CCC: Grade 0 , no visible filling of any collateral vessel; Grade 1, filling of the side branch via collateral channel but without filling of the epicardial arteries; Grade 2, partial filling of the epicardial artery via collateral branches; Grade 3, complete filling of the epicardial artery. In patients with more than 1 collateral branch, the highest grading was selected for the final analysis. The participants were then divided into good CCC group (Rentrop 2-3) and poor CCC group (Rentrop 0-1).

\section{Statistical analysis}

As is shown in Table 1, continuous variables are presented as mean \pm SD or median with interquartile range. Differences between two groups were compared by Students' t test or Mann-Whitney U test for normally or non-normally distributed continuous variates; Categorical variables are presented as frequencies and percentages whose difference between groups were performed by chi-square test; Univariate logistic regression analysis was performed to preliminarily identify potential clinical risk indicators for the poor CCC whose P value $<0.05$ was considered as clinically significant; Pearson's and Spearman's correlation analysis was used to examine the relationship between TyG index and other risk indicators. To examine the independent performance of TyG index for assessing the occurrence of poor CCC, participants were then divided into 3 groups according to TyG index tertiles (As is shown in Additional file 1). The difference between groups were compared by one way ANOVA analysis or KruskalWallis $\mathrm{H}$ test for normally or non-normally distributed variables. Difference of categorical variables between groups was compared by the chi-square test or Fisher's exact test. After adjusting for various potential risk indicators derived from univariate logistic regression analysis, a weighted multivariate logistic regression analysis was performed to calculate the odds ratios (ORs) with 95\% confidence interval (95\% Cls) of TyG index for the assessment of poor CCC. Three models constructed for this analysis were as followed: Model1 after adjustment for age, gender, BMI, smoking status, history of hypertension, T2DM, stroke and hypercholesterolemia; Model2 after adjustment for Model1 plus UREA, UA, monocyte count (MONO), hs-CRP and LVEF; Model3 after adjustment for Model2 plus severity of CAD, ISR-CTO and CTO-related artery; We further conducted a subgroup analysis through a multivariate logistic regression analysis to evaluate the association between TyG index and formation of CCC in different subgroups; Finally, to investigate the incremental value of the TyG index on the discriminative performance beyond the baseline model, ROC curves were performed to compare the AUCs. The incremental diagnostic value of TyG index for the poor CCC was examined by NRI and IDI, with the baseline model, baseline model + FPG, baseline model + GA, baseline model + TG/HDL-C and baseline 
model $+\mathrm{HbA} 1 \mathrm{c}$ as references. Statistical analysis was conducted using SPSS 23.0 (SPSS, Inc., Chicago, IL, USA) and R software (version 4.0.4). Two-sided $P$ value $<0.05$ was considered statistically significant. 
Table 1

baseline clinical and laboratory characteristics of patients

\begin{tabular}{|c|c|c|c|c|}
\hline & \multirow{2}{*}{$\begin{array}{l}\text { All Patients } \\
(n=1093)\end{array}$} & \multirow{2}{*}{$\begin{array}{l}\text { Poor CCC } \\
(n=318)\end{array}$} & \multirow{2}{*}{$\begin{array}{l}\text { Good CCC } \\
(n=775)\end{array}$} & \multirow{2}{*}{$\begin{array}{l}\mathrm{P} \\
\text { Values }\end{array}$} \\
\hline & & & & \\
\hline \multicolumn{5}{|l|}{ Demographic data } \\
\hline Age (years) & $58.76 \pm 10.17$ & $58.0 \pm 10.11$ & $59.1 \pm 10.19$ & 0.064 \\
\hline Male sex, n (\%) & $936(85.6 \%)$ & 270 (85\%) & $666(86 \%)$ & 0.659 \\
\hline $\operatorname{BMI}\left(\mathrm{Kg} / \mathrm{m}^{2}\right)$ & $26.4 \pm 3.30$ & $26.7 \pm 3.21$ & $26.2 \pm 3.32$ & 0.019 \\
\hline SBP (mmHg) & $130.1 \pm 16.49$ & $129.7 \pm 15.63$ & $130.3 \pm 16.84$ & 0.721 \\
\hline DBP (mmHg) & $77.1 \pm 11.22$ & $77.3 \pm 10.54$ & $77.0 \pm 11.49$ & 0.856 \\
\hline Current Smokers, n (\%) & 597 (55\%) & $188(59 \%)$ & 409 (53\%) & 0.056 \\
\hline \multicolumn{5}{|l|}{ Medical history } \\
\hline HTN, n (\%) & $734(67 \%)$ & $206(65 \%)$ & $528(68 \%)$ & 0.284 \\
\hline T2DM, n (\%) & $463(42 \%)$ & $147(46 \%)$ & $316(41 \%)$ & 0.098 \\
\hline Hypertriglyceridemia, n (\%) & $446(41 \%)$ & $202(64 \%)$ & $244(32 \%)$ & $\varangle 0.001$ \\
\hline Hypercholesterolemia, n (\%) & $613(56 \%)$ & $199(63 \%)$ & $414(53 \%)$ & 0.002 \\
\hline Previous MI, n (\%) & $179(16 \%)$ & $129(17 \%)$ & $50(16 \%)$ & 0.708 \\
\hline Previous Stroke, n (\%) & $109(10 \%)$ & $39(12 \%)$ & $70(9 \%)$ & 0.105 \\
\hline \multicolumn{5}{|l|}{$\begin{array}{l}\text { Number of components of } \\
\text { Mets }\end{array}$} \\
\hline 0 & $36(3 \%)$ & $6(2 \%)$ & $30(4 \%)$ & 0.095 \\
\hline 1 & $142(13 \%)$ & $26(8 \%)$ & $116(15 \%)$ & 0.002 \\
\hline 2 & $274(25 \%)$ & $59(19 \%)$ & $215(28 \%)$ & 0.001 \\
\hline 3 & $296(27 \%)$ & $95(30 \%)$ & $201(26 \%)$ & 0.183 \\
\hline 4 & $240(21 \%)$ & $85(27 \%)$ & $155(20 \%)$ & 0.015 \\
\hline 5 & $105(10 \%)$ & $47(15 \%)$ & $58(7 \%)$ & $\varangle 0.001$ \\
\hline \multicolumn{5}{|l|}{ Laboratory measurement } \\
\hline MONO, $10^{12} / \mathrm{L}$ & $0.41 \pm 0.14$ & $0.43 \pm 0.14$ & $0.40 \pm 0.14$ & 0.002 \\
\hline eGFR & $91.4 \pm 16.89$ & $91.2 \pm 18.15$ & $91.4 \pm 16.36$ & 0.693 \\
\hline CREA, mmol/L & $78.3 \pm 19.61$ & $79.2 \pm 21.28$ & $78.0 \pm 18.89$ & 0.636 \\
\hline
\end{tabular}




\begin{tabular}{|c|c|c|c|c|}
\hline & \multirow{2}{*}{$\begin{array}{l}\text { All Patients } \\
(n=1093)\end{array}$} & \multirow{2}{*}{$\begin{array}{l}\text { Poor CCC } \\
(n=318)\end{array}$} & \multirow{2}{*}{$\begin{array}{l}\text { Good CCC } \\
(n=775)\end{array}$} & \multirow{2}{*}{$\begin{array}{l}\mathrm{P} \\
\text { Values }\end{array}$} \\
\hline & & & & \\
\hline UREA, mmol/L & $5.6 \pm 1.83$ & $5.8 \pm 1.90$ & $5.5 \pm 1.80$ & 0.092 \\
\hline $\mathrm{UA}, \mathrm{mmol} / \mathrm{L}$ & $355.4 \pm 94.97$ & $369.8 \pm 97.57$ & $349.6 \pm 93.32$ & $\otimes 0.001$ \\
\hline $\mathrm{FBG}, \mathrm{mmol} / \mathrm{L}$ & $\begin{array}{l}5.88(5.11- \\
7.25)\end{array}$ & $\begin{array}{l}6.23(5.22- \\
8.10)\end{array}$ & $5.75(5.06-7.00)$ & $\bowtie 0.001$ \\
\hline $\mathrm{GA}, \%$ & $14.4(13.0-17.2)$ & $\begin{array}{l}14.5(12.9- \\
18.0)\end{array}$ & $14.4(13.0-16.8)$ & 0.455 \\
\hline $\mathrm{HbA} 1 \mathrm{C}, \%$ & $6.1(5.7-6.9)$ & $6.2(5.8-7.4)$ & $6.1(5.7-6.8)$ & 0.001 \\
\hline TyG index & $9.3 \pm 0.65$ & $9.3 \pm 0.65$ & $8.8 \pm 0.53$ & $\bowtie 0.001$ \\
\hline $\mathrm{TC}, \mathrm{mmol} / \mathrm{L}$ & $3.9 \pm 0.99$ & $4.1 \pm 1.07$ & $3.8 \pm 0.94$ & $\otimes 0.001$ \\
\hline TG, mmol/L & $1.7 \pm 0.91$ & $2.2 \pm 1.11$ & $1.6 \pm 0.73$ & $\bowtie 0.001$ \\
\hline $\mathrm{HDL}-\mathrm{C}, \mathrm{mmol} / \mathrm{L}$ & $1.0 \pm 0.25$ & $1.0 \pm 0.24$ & $1.1 \pm 0.25$ & $\varangle 0.001$ \\
\hline LDL-C, mmol/L & $2.3 \pm 0.88$ & $2.4 \pm 0.95$ & $2.2 \pm 0.85$ & 0.005 \\
\hline Non-HDL-C, mmol/L & $2.9 \pm 0.97$ & $3.1 \pm 1.05$ & $2.8 \pm 0.91$ & $\bowtie 0.001$ \\
\hline Hs CRP, mg/L & $\begin{array}{l}1.02(0.52- \\
2.64)\end{array}$ & $\begin{array}{l}1.13(0.68- \\
3.16)\end{array}$ & $\begin{array}{l}0.97(0.48- \\
2.38)\end{array}$ & 0.002 \\
\hline LVEF, \% & $59.3 \pm 8.59$ & $59.0 \pm 8.91$ & $59.5 \pm 8.46$ & 0.343 \\
\hline \multicolumn{5}{|l|}{ Cardiovascular Medication } \\
\hline Antiplatelet therapy, n (\%) & $940(86 \%)$ & $274(86 \%)$ & $666(86 \%)$ & 0.921 \\
\hline ß-blockers, n (\%) & $660(60 \%)$ & $200(63 \%)$ & $460(59 \%)$ & 0.277 \\
\hline ACEI/ARB, n (\%) & $360(33 \%)$ & $112(35 \%)$ & $248(32 \%)$ & 0.304 \\
\hline Lipid-lowering drugs, n (\%) & $902(83 \%)$ & 267 (84\%) & $635(82 \%)$ & 0.423 \\
\hline CCB, n (\%) & $305(28 \%)$ & $80(25 \%)$ & $225(29 \%)$ & 0.195 \\
\hline Severity of CAD & & & & 0.912 \\
\hline One-vessel disease, $\mathrm{n}(\%)$ & $154(14 \%)$ & $43(14 \%)$ & $111(14 \%)$ & 0.775 \\
\hline Two-vessel disease, n (\%) & $313(29 \%)$ & $90(28 \%)$ & $223(29 \%)$ & 0.941 \\
\hline $\begin{array}{l}\text { LM/Three-vessel disease, } \\
\text { n (\%) }\end{array}$ & $626(57 \%)$ & $185(58 \%)$ & $441(57 \%)$ & 0.737 \\
\hline CTO related artery & & & & 0.078 \\
\hline RCA & $528(48 \%)$ & 139 (44\%) & $389(50 \%)$ & 0.054 \\
\hline
\end{tabular}




\begin{tabular}{|c|c|c|c|c|}
\hline & All Patients & Poor CCC & & $P$ \\
\hline & $(n=1093)$ & $(n=318)$ & $(n=775)$ & \\
\hline LCX & 192 (18\%) & $55(17 \%)$ & 137 (18\%) & 0.93 \\
\hline LAD & 373 (34\%) & 124 (39\%) & 249 (32\%) & 0.035 \\
\hline Grade of $c$ & & & & \\
\hline 0 & 69 (6\%) & 69 (22\%) & - & \\
\hline 1 & $249(23 \%)$ & 249 (78\%) & - & \\
\hline 2 & $551(50 \%)$ & - & $551(71 \%)$ & \\
\hline 3 & $224(21 \%)$ & - & $224(29 \%)$ & \\
\hline ISR-CTO & $104(10 \%)$ & 37 (12\%) & 67 (9\%) & 0.14 \\
\hline $\begin{array}{l}\text { BMI body } \\
\text { T2DM typ } \\
\text { MONO mc } \\
\text { FPG fastir } \\
\text { triglycerid } \\
\text { cholesterc } \\
\text { cholesterc } \\
\text { angiotens } \\
\text { blocker, C/ } \\
\text { circumflex }\end{array}$ & $\begin{array}{l}\text { lic blood press } \\
\mathrm{Cl} \text { percutaneo } \\
\text { d glomerular f } \\
\text { glycated albur } \\
\text { al cholesterol, } \\
\text { oprotein chole } \\
\text { ity C-reactive p } \\
\text { hhibitors / ang } \\
\text { ase, CTO chro } \\
\text { eft anterior des }\end{array}$ & $\begin{array}{l}\text { BP diastolic } \\
\text { ronary interve } \\
\text { on rate, CREA } \\
\text { tbA1c glycos } \\
\text { glyceride, HD } \\
\text { l, non HDL-C r } \\
\text { l, LVEF left ve } \\
\text { sin receptor b } \\
\text { tal lesion, RC } \\
\text { ing artery ISR }\end{array}$ & $\begin{array}{l}\text { pressure, HTN } \\
\text { MetS metabo } \\
\text { ine, UREA ure } \\
\text { hemoglobin A } \\
\text { h-density lipo } \\
\text { h-density lipc } \\
\text { ar ejection fra } \\
\text { s, CCB calciun } \\
\text { coronary arte } \\
\text { th restenosis }\end{array}$ & $\begin{array}{l}\text { tension, } \\
\text { drome, } \\
\text { uric acid, } \\
\text { G index } \\
\text { n } \\
\text { ACEI/ARB } \\
\text { nel } \\
\text { left }\end{array}$ \\
\hline
\end{tabular}

\section{Results}

\section{Baseline Characteristics}

The demographic, laboratory and angiographic characteristics of participants were shown in Table 1. They were divided into good CCC group $(n=775)$ and bad CCC group $(n=318)$ according to the Rentrop scoring system. There was no difference in age and proportion of sex between two groups, but most of them were males. Compared with those with good CCC, those with poor collateralization had higher BMI and higher rates of hyperlipidemia; Furthermore, though not very statistically significant, poor CCC groups were more likely to be cigarette smokers and T2DM patients. No difference was found in the history of hypertension, stroke, $\mathrm{MI}$, past $\mathrm{PCl}$ and taking cardiovascular medications between two groups. Biochemical measurements showed that patients with good CCC have lower lipid profiles, FPG, HbA1c, TyG index, UA, a tendency of lower UREA and lower inflammatory status. Our analysis found no difference between two groups in eGFR, creatine (CREA), GA and LVEF. Coronary angiographic analysis indicated that the incidences of CTO in right coronary artery (RCA), left circumflex artery (LCX) and left anterior artery (LAD) were $48 \%, 18 \%$ and $34 \%$ respectively, with a difference of $P=0.078$ between two groups. The distribution of number of the diseased vessels shows no significantly different $(P=0.912)$, as patients with one-vessel disease, two-vessel disease and multi-vessel disease were $14 \%$ vs. $14 \%(P=$ 
$0.775), 28 \%$ vs. $29 \%(P=0.941)$ and $58 \%$ vs. $57 \%(P=0.737)$ in the poor CCC and good CCC groups. It seems that RCA-related CTO lesions tends to have good CCC ( $50 \%$ vs. $44 \%, P=0.054)$, while CTO lesions located in LAD are likely to have poor CCC (39\% vs. $32 \%, P=0.035)$; no significant difference of LCXrelated CTO lesions was observed in this study. Notably, the rate of poor CCC seemed to be higher in patients whose CTO lesions caused by In-Stent Restenosis (ISR) (12\% vs. 9\%, P $=0.140)$

Table 2 showed that TyG index is significantly associated with some laboratory and demographic measurements by using Pearson's and Spearman's correlation analysis. Positive correlation was found between the TyG index and the components of MetS, BMI, lipid profiles, glucose metabolism markers, UA and hs-CRP, while negative correlation was indicated with age, HDL-C and eGFR; We find the correlation between TyG index and MetS was the strongest among those factors $(r=0.624, P<0.001)$.

Table 2

Correlation between TyG index and recognized cardiovascular risk factors

\begin{tabular}{|c|c|c|}
\hline & Correlation coefficient & P values \\
\hline Age & -0.067 & 0.027 \\
\hline Gender & 0.043 & 0.152 \\
\hline BMI & 0.193 & $\varangle 0.001$ \\
\hline TC & 0.305 & $\llbracket 0.001$ \\
\hline LDL-C & 0.182 & $\bigotimes 0.001$ \\
\hline HDL-C & -0.314 & $\llbracket 0.001$ \\
\hline Non-HDL-C & 0.393 & $\llbracket 0.001$ \\
\hline $\mathrm{HbA} 1 \mathrm{C}$ & 0.387 & $\bigotimes 0.001$ \\
\hline GA & 0.301 & $\bigotimes 0.001$ \\
\hline UA & 0.176 & $\bigotimes 0.001$ \\
\hline eGFR & -0.073 & 0.015 \\
\hline hs-CRP & 0.08 & 0.008 \\
\hline LVEF & 0.012 & 0.71 \\
\hline Number of components of Mets & 0.624 & $\llbracket 0.001$ \\
\hline \multicolumn{3}{|c|}{$\begin{array}{l}\text { BMI body mass index, TC total cholesterol, LDL-C low-density lipoprotein cholesterol, HDL-C high- } \\
\text { density lipoprotein cholesterol, non-HDL-C non-high-density lipoprotein cholesterol, HbA1c } \\
\text { glycosylated hemoglobin A1c, GA glycated albumin, eGFR estimated glomerular filtration rate, UA uric } \\
\text { acid, hs-CRP high-sensitivity C-reactive protein, LVEF left ventricular ejection fraction, MetS metabolic } \\
\text { syndrome }\end{array}$} \\
\hline
\end{tabular}




\section{Association Between Tyg Index And Ccc}

As is shown in Fig. 2A, TyG index in patients with poor collateralization was significantly higher than those with good one $(9.3 \pm 0.65$ vs. $8.8 \pm 0.53, P<0.001)$. Once dividing these participants into 3 tertiles according to the TyG index, the proportion of poor collateralization increased stepwise from the lowest TyG index tertile to the highest one ( $15.3 \%$ vs. $22.8 \%$ vs. $49.2 \%, \mathrm{P}<0.001)$ (Fig. 2B). Multivariate logistic regression analysis was performed upon division, Table 3 manifested the odds ratios (ORs) and 95\% confidence intervals (Cls) for poor CCC based on the TyG index tertiles. Unadjusted logistic regression analysis was performed to initially test the ORs of TyG index for the poor CCC, the ORs ( $95 \% \mathrm{Cls}$ ) of TyG index for the poor CCC in T2 and T3 group versus T1 group were 1.63 (1.12-2.37) and 5.34 (3.76-7.58), respectively. Three models were constructed to perform adjusted logistic regression analysis: Model1, after adjustment for age, sex, BMI, smoking history, Stroke, hypertension, T2DM and hypercholesterolemia; Model2, after adjustment for Model1 plus MONO, UREA, hs-CRP and LVEF; Model3, after adjustment for Model 2 plus number of diseased vessels, CTO-related artery and ISR-CTO; The values of ORs ( $95 \% \mathrm{Cls})$ of T2 and T3 groups versus T1 were shown in Table 3. The factors influencing the formation of CCC were shown in Fig. 3 after multivariable logistic regression analysis. Figure 4 presented the results of subgroup analysis showing the relationship between TyG index and CCC in populations with different clinical characteristics. The adjusted ORs of TyG index for evaluating CCC remained significant across all subgroups. Finally, Additional file 2 showed the incremental diagnostic value of TyG index on the baseline model consisting of recognized risk factors related to poor CCC and its performance are better than $\mathrm{HbA} 1 \mathrm{C}$ and TG/HDL-C. The improvement of AUC for assessing the poor CCC was most significant when adding TyG index to the baseline model with a best cut-off value of 9.105 for evaluating poor CCC (Fig. 5) (AUC: baseline model 0.629 vs. +TyG index 0.724; baseline model 0.629 vs. +FPG 0.673 ; baseline model 0.629 vs. +GA 0.632; baseline model 0.629 vs. $+\mathrm{HbA} 1 \mathrm{c} 0.638$; baseline model 0.629 vs. + TG/HDL-C 0.721). Furthermore, the most significant enhancements on risk reclassification and discrimination were found after inclusion the TyG index into baseline model, with a NRI of $0.238(\mathrm{P}<$ $0.001)$ and an IDI of $0.103(P<0.001)$, when compared with FPG (NRI, 0.067, P = 0.009; IDI, 0.037, $P<$ $0.001)$ and TG/HDL-C (NRI, 0.159, P<0.001; IDI, 0.087, P<0.001) (Table 4) 
Table 3

Association between TyG index and poor CCC

\begin{tabular}{|c|c|c|c|c|c|}
\hline Tyg index & Sample size of poor CCC, $n$ (\%) & ORs & $95 \% \mathrm{Cl}$ & $\mathbf{P}$ & P for trend \\
\hline Unadjusted & & & & & $₫ 0.001$ \\
\hline T1 & 56 & Ref. & 1 (Ref.) & Ref. & \\
\hline T2 & 83 & 1.63 & $1.12-2.37$ & 0.011 & \\
\hline T3 & 179 & 5.34 & $3.76-7.58$ & $\varangle 0.001$ & \\
\hline Model 1 & & & & & $\varangle 0.001$ \\
\hline T1 & 56 & Ref. & 1 (Ref.) & Ref. & \\
\hline $\mathrm{T} 2$ & 83 & 1.67 & $1.13-2.46$ & 0.010 & \\
\hline T3 & 179 & 5.62 & $3.91-8.29$ & $\varangle 0.001$ & \\
\hline Model 2 & & & & & $₫ 0.001$ \\
\hline T1 & 56 & Ref. & 1 (Ref.) & Ref. & \\
\hline T2 & 83 & 1.59 & $1.08-2.35$ & 0.020 & \\
\hline T3 & 179 & 5.31 & $3.58-7.87$ & $\varangle 0.001$ & \\
\hline Model 3 & & & & & $\varangle 0.001$ \\
\hline T1 & 56 & Ref. & 1 (Ref.) & Ref. & \\
\hline T2 & 83 & 1.59 & $1.07-2.36$ & 0.021 & \\
\hline T3 & 179 & 5.72 & $3.83-8.54$ & $\nabla 0.001$ & \\
\hline
\end{tabular}

Model 1: adjustment for age, sex, BMI, Smoking status, previous Stroke, Hypertension, Hypercholesterolemia, T2DM;

Model2: adjustment for Model1 + MONO + UREA + UA + hsCRP + LVEF

Model3: adjustment for Model2 + severity of CAD + ISR-CTO + CTO related artery;

TyG index triglyceride glucose index, CCC coronary collateral circulation, OR odds ratio, $\mathrm{Cl}$ confidence interval, BMI body mass index, HTN hypertension, T2DM type 2 diabetes mellitus, MONO monocyte, UREA urea, UA uric acid, hs-CRP high sensitivity C-reactive protein, LVEF left ventricular ejection fraction, CAD coronary artery disease, ISR In-stent restenosis, CTO chronic total lesion,

\section{Tyg Index And Metabolic Syndrome}

In order to substantiate the relationship between TyG index and MetS. Spearman correlation analysis was performed Table 2. Besides, the association between the TyG index and the MetS was assessed by using the MetS as a quantitative data (from 0 to 5 according to the number of components of the MetS), the 
value of TyG index significantly increases with the number of the components of MetS, from $8.27 \pm 0.34$ (component of the MetS =0) to $9.68 \pm 0.44$ (component of the MetS = 5), with a P value $<0.001$.

\section{Discussion}

The major findings of this study are as follows: TyG index was significantly lower in patients with good CCC than those with poor one. TyG index was associated with various traditional cardiovascular risk factors, a strongest correlation was found between TyG index and components of MetS. Multiple logistic regression analysis and subgroup analysis showed that TyG index remains to be strongest risk indicator of coronary collateralization after adjustment for various confounding factors. Its risk assessment value was better than traditional lipid or glucose related parameters, like TG, FPG and HbA1c. Adding TyG index to the baseline model shows the most significant incremental effect on the risk discrimination for assessing the development of coronary collateral growth. To the best of our knowledge, this is the first study focusing on the correlation of TyG index and coronary collateralization.

\section{Impact Of Ir On Impaired Coronary Collateralization}

Coronary collateralization is a network of anastomose connecting epicardial coronary branches from different regions within heart, which conduct little blood flow to produce cardioprotection because of its small caliber and high resistance in physiological conditions. However, when epicardial arteries were severely obstructed, especially in those with chronic total occlusion, those anastomoses can turn into functional branch collaterals to compensate for the blood supply to the myocardial regions dominated by the stenotic arteries. In ideal circumstances, a well-developed coronary collaterals can perform myocardial salvaging effect to limit the ischemic area caused by CAD and preserve normal cardiac function[5]. previous study found that the coronary collateral could expand four times bigger from a caliber of $10-200 \mu \mathrm{m}$ to $100-800 \mu \mathrm{m}$ in the presence of CAD[25]. Mechanism underlying the formation of functional collateral network in adult animals mainly involves the abluminal expansion and wall thickening of preexisting one called coronary collateral growth (CCG), also termed as arteriogenesis.[26] Although the mechanism of arteriogenesis remained to be incompletely understood, clinical evidence has shown that CCG is impaired in patients with MetS[10,27]. The current study revealed that TyG index, as a simple surrogate index of IR, is independently associated with less developed CCC, indicating that IR may play a pivotal role in the development of collateral circulation. IR, as a key parameter of MetS, affected factors that impaired the development of CCG, such as decreased expression of proangiogenic growth factors, increased production of reactive oxidative species (ROS) and continued endothelial dysfunction. The complete obstruction of coronary artery leads to the elevated pressure gradient of arterioles and therefore increasing tangential fluid shear stress (FSS) to activate monocytes to release some angiogenic growth factors, like MCP-1, TGF- $\beta[28]$. Recent study has shown that monocyte-derived dendritic cells (MoDCs) tend to be functionally defective in the context of IR and thus increasing vascular inflammation, which would deteriorate the development of collateral growth[29]. Overproduction of ROS is another reason for less developed CCC. IR-induced hyperglycemia leads to the increase of polyol (sorbitol) 
pathway influx, advanced glycation end-product (AGE) production, hexosamine pathway influx and the activation of protein kinase $\mathrm{C}$ (PKC) and thus causing the hyperglycemia-induced overproduction of ROS by the activating those pathways and the mitochondrial electron-transport chain[30]. People with IR are characterized by the impairment of endothelial function and IR was inversed associated with median colony forming unit endothelial cells (CFU-ECs), causing the decreased density of collaterals in response of cardiac ischemia[10]. Recent study has revealed that IR could decrease the expression of MicroRNA-21 (MiR-21), an important mediator to regulate the secretion of NO and ET-1, via the inhibition of PTEN/AKT/eNOS pathway and activation of MAPK/ET-1 pathway and thereby causing endothelial dysfunction[31]. While present study provided clinical evidence for evaluating the impact of IR on collateral growth, more clinical and basic researches are still needed to clarify the underlying mechanisms of IR-induced poor collateralization.

\section{Relationship Between Tyg Index And Coronary Collateral Circulation}

To the best of our knowledge, this is the first study to show that the TyG index is strongly associated with the development of $\mathrm{CCC}$ even after adjusting for various confounding factors. Several recent cohort studies about TyG index in predicting long-term cardiovascular outcomes in patients with ACS have been published in succession. Mao et. al[17] for the first time proved that TyG index is independently related to coronary artery disease severity and could act as a strong predictor of MACEs in patients with non-STsegment elevation acute coronary syndrome (NSTE-ACS) after a 12-month follow-up. However, the conclusion of this study was easily influenced by relatively small sample sizes and short follow-up period. Besides, this study also failed to take acute myocardial infarction (AMI) patients into consideration. A cohort study conducted by Zhang et. al[19] tried to substantiate this conclusion in AMI patients with T2DM and found TyG index is positively associated with cardiovascular death, non-fatal MI, cardiac rehospitalization, revascularization and composite MACEs with the optimal cut-off value of 9.30, which suggested its potential role in risk stratification and prediction of prognosis in patients with AMI and T2DM. Besides, its predictive value was also applicable for those ACS patients undergoing $\mathrm{PCl}$. A study of 776 ACS patients accepting PCI showed that TyG index could be a valuable predictor of cardiovascular outcomes after PCl in ACS patients with T2DM[32]. Another cohort study consisting of 2531 ACS patients undergoing non-invasive or invasive therapy with a 3-year follow-up also showed that the incidence of MACE, defined as all-cause death, non-fatal myocardial infarction and non-fatal stroke increased with the increasing TyG index tertiles with an optimal cut-off value of 9.323 and TyG index had an incremental value on the prognostic model for predicting MACE, but subgroup analysis failed to show that TyG index was an independent predictor for MACE in NSTEMI and STEMI subgroups[16]. In addition, the prognostic value of TyG index in nondiabetic ACS patients was also assessed. Zhang et. al found in ACS patients with LDL-C below $1.8 \mathrm{mmol} / \mathrm{L}$,high TyG index was associated with increasing incidence of AMI, larger infarct size and increasing revascularization rates[18]. Although the mechanism underlying the association of TyG index with adverse cardiovascular outcomes has not been fully elucidated, 
considering the beneficial role of CCC in preventing cardiac ischemia and further myocardial infarction, the present study indicates that CAD patients with prominent coronary stenosis whose TyG index $\geq 9.11$ tend to form less developed $\mathrm{CCC}$ and at least provides a new clinical insight into understanding the role of TyG index in predicting the long-term prognosis of CAD patients.

Subgroup analysis of this study showed that the association between TyG index and coronary collateralization remained significant across all subgroups, but we failed to substantiate significant interaction between TyG index and HTN and inflammatory status for evaluating the formation of CCC, considering hypertension and inflammation having impact on the coronary collateralization in precious studies, more researches are needed to understand the mechanism underlying the development of coronary collateralization.

Apart from showing the independent relationship between the TyG index and poor collateralization, the current study also found that adding TyG index to the baseline risk models had a significantly higher incremental effect on the risk assessment value for the formation of CCC than FPG, TG and another IR surrogate marker TG/HDL-C, indicating that TyG index would be more valuable in evaluating the incidence of poor CCC beyond TG, FPG and TG/HDL-C. In addition, Fig. 5 showed the AUC of baseline model, baseline model + TG/HDL-C, baseline model + HbA1c and baseline model + TyG index for assessing the incidence of poor $\mathrm{CCC}$, a significant improvement on risk discriminative efficacy was found when adding TyG index into the baseline model when compared with TG/HDL-C and HbA1c. Several recent studies have compared the predictive value of TyG index, FPG and HbA1c for cardiovascular events in ACS patients and attained positive results for TyG index[33-35]. Discordance analysis conducted by Hu et. al[34] indicated that high TyG index was always associated with relatively high risk of cardiovascular events in ACS patients accepting PCI when dividing those patients into different groups based on low/high FPG or HbA1c categories, highlighting its role of better predicting cardiovascular risks than FPG or HbA1c. Better predictive value for MACCE were also substantiated when adding TyG index into baseline risk model than FPG or HbA1c in AMI patients with diabetes (AUC 0.685 for baseline model + TyG index vs. 0.661 for baseline model + HbA1c vs. 0.664 for baseline model + FPG, $P<0.001$; NRI $0.190, P<0.001$; IDI 0.027, $P<0.001$ ) and NSTE-ACS patients with non-diabetes (AUC 0.853 for baseline model + TyG index vs. 0.835 for baseline model + HbA1c vs. 0.837 for baseline model + FPG, $P<0.001$; NRI 0.194, $P<0.001$; IDI 0.023, $P<0.001$ ][35]. Considering the relatively easier access to acquire and more accurately predictive value for cardiovascular events than traditional diabetic status marker, such as TG/HDL-C or HbA1c, our study would provide more evidence for putting TyG index into clinical practice to identify patients with high cardiovascular risks, especially for those without diabetes.

\section{Correlation Between Tyg Index And Mets}

Given the high prevalence of MetS in CAD patients, there's a need to develop convenient and low-cost screening tools to predict MetS and evaluate long-term cardiovascular risks in those MetS-related CAD patients. Our current study found the strongest link between MetS and TyG index $(r=0.624, P<0.001)$ by 
using Spearman's correlation analysis and the value of TyG index increases with the number of components of MetS. This is the first study to evaluate the association between TyG index and the components of MetS in ACS patients, especially in those with complicated coronary lesions. Recent studies have shown the efficacy of using TyG index to identify MetS. A population-based study containing 5000 participants found TyG index exhibited the best performance value in identifying MetS among obesity-related indices[36]. A large cross-sectional study from Korea also found the best cut-off value of TyG index for predicting MetS in middle-aged and older populations was 8.81 and they also showed the value of TyG index increased stepwise with the increasing components of MetS[37], which is in accordance with our current findings. In addition, TyG index could also predict the potential of developing MetS in healthy populations. A cohort study conducted by Lin et. al[12] showed that subjects with higher TyG index are more likely to develop MetS in healthy populations over 5-year follow-up and its predictive value was better than that of blood leukocyte indices, with an optimal cut-off value of 8.52 . The current study would provide clinical evidence for using TyG index as an efficient tool to evaluate MetS in ACS patients, suggesting a therapeutic potential of TyG index in treating cardiometabolic diseases. However, given current studies mainly focused on evaluating TyG index and MetS in general population, more researches are needed to assess TyG index and MetS in patients with CAD.

\section{Limitations}

Several limitations should be acknowledged. First, the nature of single-center study may invariably involve some selection bias. Second, this cross-sectional observational study can only allow us to detect the strong association between TyG index and CCC but incapable of assessing the casual link between them. Third, the grading and evaluation of CCC in our study were based on Rentrop scoring system because it's easy to operate in clinical practice. However, coronary collaterals should be more accurately assessed by diagnostic gold standard like CFI. Besides, there was no gold standard test to evaluate IR, so we could not compare TyG index with other IR index for assessing CCC and thus limiting its generalization use. Lastly, TyG index is easily affected by many factors, the population in our current study is mainly ACS patients, including those experiencing AMI, which would cause acute fluctuation in

glucose levels and thus affecting TyG index values. TyG index is also affected by drugs that influence the level of TG, however, in this study, we generally used the history of taking any lipid-lowering drugs and failed to take TG-lowering drugs into our analysis. Therefore, further researches could be conducted on the impact of TG-lowering drugs like fibrates on the formation of CCC.

\section{Conclusion}

In conclusion, TyG index is strongly associated with the development of coronary collaterals even after adjusting for various confounding factors and its risk discriminative value are better than $\mathrm{HbA} 1 \mathrm{c}$ and another IR surrogate index, like TG/HDL-C.

\section{Abbreviations}


TyG index: triglyceride glucose index; CCC: coronary collateral circulation; ACS: acute coronary syndrome; NSTE-ACS: non-ST-segment elevation acute coronary syndrome; AMl: acute myocardial infarction; UAP: unstable angina pectoris; NSTEMI: Non-ST Elevated Myocardial Infarction; STEMI: ST Elevated Myocardial Infarction; TIMI: Thrombolysis in Myocardial Infarction; CTO: chronic total occlusion; PCI: percutaneous coronary intervention; CABG: coronary artery bypass grafting; HbA1 c: glycosylated hemoglobin A1c; IR: insulin resistance; ROC: receiver-operating characteristic; AUC: area under curve; NRI: net reclassification improvement; IDI: integrated discrimination improvement; MACE: major adverse cardiovascular events; CKD: chronic kidney disease; ISR: In-Stent Restenosis; MetS: metabolic syndrome; HOMA-IR: homeostasis model assessment of insulin resistance; T2DM: type 2 mellitus diabetes; eGFR: estimated glomerular filtration rate; SBP: systolic blood pressure; DBP: diastolic blood pressure; TC: total cholesterol; LDL-C: low-density lipoprotein cholesterol; TG: triglycerides; HDL-C: high-density lipoprotein cholesterol; non-HDL-C: non-high-density lipoprotein cholesterol; BMI: body mass index; LVEF: left ventricular ejection fraction; FPG: fasting plasma glucose; GA: glycosylated albumin; UA: uric acid; hsCRP: high-sensitivity C-reactive protein; CAG: coronary angiography; HGB: hemoglobin; MONO: monocyte count; RCA: right coronary artery; LCX: left circumflex artery; LAD: left anterior artery; CCG: coronary collateral growth; ROS: reactive oxidative species; FSS: fluid shear stress; Mo-DCs: monocyte-derived dendritic cells; AGE: advanced glycation end-product; OGTT: 75g oral glucose tolerance test;

\section{Declarations}

\section{Availability of data and materials}

The datasets and materials mentioned above are available from the authors.

\section{Ethics approval and consent to participate}

The current study was conducted in accordance with Declaration of Helsinki and was approved by the Ethics Committee of Beijing Anzhen Hospital, Capital Medical University. Given the retrospective nature of this study, the informed consent from participants was waived.

\section{Competing interests}

The authors declare that they have no competing interests

\section{Consent for publications}

Not applicable.

\section{Authors' contributions}

YZ and AG contributed to the conception and design of this study and AG wrote this article. All authors made contribution to collect and analyze data. All authors read and approved the final manuscript. 


\section{Authors' details}

${ }^{1}$ Department of Cardiology, Beijing Anzhen Hospital, Capital Medical University, Beijing 100029, China.

2Beijing Institute of Heart Lung and Blood Vessel Disease, Beijing 100029, China.

\section{References}

1. Yang Q, Wang Y, Liu J, Liu J, Hao Y, Smith SC Jr, Huo Y, Fonarow GC, Ma C, Ge J, et al: Invasive Management Strategies and Antithrombotic Treatments in Patients With Non-ST-Segment-Elevation Acute Coronary Syndrome in China: Findings From the Improving CCC Project (Care for Cardiovascular Disease in China). Circ Cardiovasc Interv 2017, 10.

2. Gallone G, Baldetti L, Tzanis G, Gramegna M, Latib A, Colombo A, Henry TD, Giannini F. Refractory Angina: From Pathophysiology to New Therapeutic Nonpharmacological Technologies. JACC Cardiovasc Interv. 2020;13:1-19.

3. Brilakis ES, Banerjee S, Karmpaliotis D, Lombardi WL, Tsai TT, Shunk KA, Kennedy KF, Spertus JA, Holmes DR Jr, Grantham JA. Procedural outcomes of chronic total occlusion percutaneous coronary intervention: a report from the NCDR (National Cardiovascular Data Registry). JACC Cardiovasc Interv. 2015;8:245-53.

4. Lee SP, Shin DH, Park KW, Kang HJ, Koo BK, Cho YS, Yeon TJ, Chae IH, Choi DJ, Kim HS.

Angiographic patterns of restenosis after percutaneous intervention of chronic total occlusive lesions with drug-eluting stents. Int J Cardiol. 2012;156:180-5.

5. Seiler C, Stoller M, Pitt B, Meier P. The human coronary collateral circulation: development and clinical importance. Eur Heart J. 2013;34:2674-82.

6. Elias J, Hoebers LPC, van Dongen IM, Claessen B, Henriques JPS. Impact of Collateral Circulation on Survival in ST-Segment Elevation Myocardial Infarction Patients Undergoing Primary Percutaneous Coronary Intervention With a Concomitant Chronic Total Occlusion. JACC Cardiovasc Interv. 2017;10:906-14.

7. Hara M, Sakata Y, Nakatani D, Suna S, Nishino M, Sato H, Kitamura T, Nanto S, Hori M, Komuro I, Investigators $\mathrm{O}$. Impact of coronary collaterals on in-hospital and 5-year mortality after ST-elevation myocardial infarction in the contemporary percutaneous coronary intervention era: a prospective observational study. BMJ Open. 2016;6:e011105.

8. Epstein SE, Lassance-Soares RM, Faber JE, Burnett MS. Effects of aging on the collateral circulation, and therapeutic implications. Circulation. 2012;125:3211-9.

9. Rocic P. Why is coronary collateral growth impaired in type II diabetes and the metabolic syndrome? Vascul Pharmacol. 2012;57:179-86.

10. Mouquet F, Cuilleret F, Susen S, Sautiere K, Marboeuf P, Ennezat PV, McFadden E, Pigny P, Richard F, Hennache B, et al. Metabolic syndrome and collateral vessel formation in patients with documented 
occluded coronary arteries: association with hyperglycaemia, insulin-resistance, adiponectin and plasminogen activator inhibitor-1. Eur Heart J. 2009;30:840-9.

11. Lim J, Kim J, Koo SH, Kwon GC. Comparison of triglyceride glucose index, and related parameters to predict insulin resistance in Korean adults: An analysis of the 2007-2010 Korean National Health and Nutrition Examination Survey. PLoS One. 2019;14:e0212963.

12. Lin HY, Zhang XJ, Liu YM, Geng LY, Guan LY, Li XH. Comparison of the triglyceride glucose index and blood leukocyte indices as predictors of metabolic syndrome in healthy Chinese population. Sci Rep. 2021;11:10036.

13. Wen J, Wang A, Liu G, Wang M, Zuo Y, Li W, Zhai Q, Mu Y, Gaisano HY, He Y, Dou J. Elevated triglyceride-glucose (TyG) index predicts incidence of Prediabetes: a prospective cohort study in China. Lipids Health Dis. 2020;19:226.

14. Park K, Ahn CW, Lee SB, Kang S, Nam JS, Lee BK, Kim JH, Park JS. Elevated TyG Index Predicts Progression of Coronary Artery Calcification. Diabetes Care. 2019;42:1569-73.

15. Lee SB, Ahn CW, Lee BK, Kang S, Nam JS, You JH, Kim MJ, Kim MK, Park JS. Association between triglyceride glucose index and arterial stiffness in Korean adults. Cardiovasc Diabetol. 2018;17:41.

16. Wang L, Cong HL, Zhang JX, Hu YC, Wei A, Zhang YY, Yang H, Ren LB, Qi W, Li WY, et al. Triglycerideglucose index predicts adverse cardiovascular events in patients with diabetes and acute coronary syndrome. Cardiovasc Diabetol. 2020;19:80.

17. Mao Q, Zhou D, Li Y, Wang Y, Xu SC, Zhao XH. The Triglyceride-Glucose Index Predicts Coronary Artery Disease Severity and Cardiovascular Outcomes in Patients with Non-ST-Segment Elevation Acute Coronary Syndrome. Dis Markers. 2019;2019:6891537.

18. Zhang Y, Ding X, Hua B, Liu Q, Gao H, Chen H, Zhao XQ, Li W, Li H. High Triglyceride-Glucose Index is Associated with Poor Cardiovascular Outcomes in Nondiabetic Patients with ACS with LDL-C below $1.8 \mathrm{mmol} / \mathrm{L}$. J Atheroscler Thromb 2021.

19. Zhang Y, Ding X, Hua B, Liu Q, Gao H, Chen H, Zhao XQ, Li W, Li H. High triglyceride-glucose index is associated with adverse cardiovascular outcomes in patients with acute myocardial infarction. Nutr Metab Cardiovasc Dis. 2020;30:2351-62.

20. Brilakis ES, Mashayekhi K, Tsuchikane E, Abi Rafeh N, Alaswad K, Araya M, Avran A, Azzalini L, Babunashvili AM, Bayani B, et al. Guiding Principles for Chronic Total Occlusion Percutaneous Coronary Intervention. Circulation. 2019;140:420-33.

21. American Diabetes A. 2. Classification and Diagnosis of Diabetes: Standards of Medical Care in Diabetes-2018. Diabetes Care. 2018;41:13-27.

22. Williams B, Mancia G, Spiering W, Agabiti Rosei E, Azizi M, Burnier M, Clement DL, Coca A, de Simone G, Dominiczak A, et al. $2018 \mathrm{ESC} / \mathrm{ESH}$ Guidelines for the management of arterial hypertension. Eur Heart J. 2018;39:3021-104.

23. Executive Summary of The Third Report of The National Cholesterol Education Program (NCEP) Expert Panel on Detection, Evaluation, And Treatment of High Blood Cholesterol In Adults (Adult Treatment Panel III). Jama 2001, 285:2486-2497. 
24. Fan L, Hao Z, Gao L, Qi M, Feng S, Zhou G. Non-linear relationship between sleep duration and metabolic syndrome: A population-based study. Med (Baltim). 2020;99:e18753.

25. Fulton WF. ARTERIAL ANASTOMOSES IN THE CORONARY CIRCULATION. II. DISTRIBUTION, ENUMERATION AND MEASUREMENT OF CORONARY ARTERIAL ANASTOMOSES IN HEALTH AND DISEASE. Scott Med J. 1963;8:466-74.

26. Carmeliet P. Mechanisms of angiogenesis and arteriogenesis. Nat Med. 2000;6:389-95.

27. Liu T, Wu Z, Liu J, Lv Y, Li W. Metabolic syndrome and its components reduce coronary collateralization in chronic total occlusion: An observational study. Cardiovasc Diabetol. 2021;20:104.

28. Rubanyi GM. Mechanistic, technical, and clinical perspectives in therapeutic stimulation of coronary collateral development by angiogenic growth factors. Mol Ther. 2013;21:725-38.

29. Paccosi S, Pala L, Cresci B, Silvano A, Cecchi M, Caporale R, Maria Rotella C, Parenti A. Insulin resistance and obesity affect monocyte-derived dendritic cell phenotype and function. Diabetes Res Clin Pract. 2020;170:108528.

30. Papachristoforou E, Lambadiari V, Maratou E, Makrilakis K. Association of Glycemic Indices (Hyperglycemia, Glucose Variability, and Hypoglycemia) with Oxidative Stress and Diabetic Complications. J Diabetes Res. 2020;2020:7489795.

31. Liu R, Guan S, Gao Z, Wang J, Xu J, Hao Z, Zhang Y, Yang S, Guo Z, Yang J, et al. Pathological Hyperinsulinemia and Hyperglycemia in the Impaired Glucose Tolerance Stage Mediate Endothelial Dysfunction Through miR-21, PTEN/AKT/eNOS, and MARK/ET-1 Pathways. Front Endocrinol (Lausanne). 2021;12:644159.

32. Ma X, Dong L, Shao Q, Cheng Y, Lv S, Sun Y, Shen H, Wang Z, Zhou Y, Liu X. Triglyceride glucose index for predicting cardiovascular outcomes after percutaneous coronary intervention in patients with type 2 diabetes mellitus and acute coronary syndrome. Cardiovasc Diabetol. 2020;19:31.

33. Su WY, Chen SC, Huang YT, Huang JC, Wu PY, Hsu WH, Lee MY. Comparison of the Effects of Fasting Glucose, Hemoglobin A1c, and Triglyceride-Glucose Index on Cardiovascular Events in Type 2 Diabetes Mellitus. Nutrients 2019, 11.

34. Hu C, Zhang J, Liu J, Liu Y, Gao A, Zhu Y, Zhao Y. Discordance between the triglyceride glucose index and fasting plasma glucose or $\mathrm{HbA} 1 \mathrm{C}$ in patients with acute coronary syndrome undergoing percutaneous coronary intervention predicts cardiovascular events: a cohort study from China. Cardiovasc Diabetol. 2020;19:116.

35. Zhao Q, Zhang TY, Cheng YJ, Ma Y, Xu YK, Yang JQ, Zhou YJ. Triglyceride-Glucose Index as a Surrogate Marker of Insulin Resistance for Predicting Cardiovascular Outcomes in Nondiabetic
Patients with Non-ST-Segment Elevation Acute Coronary Syndrome Undergoing Percutaneous
Coronary Intervention. J Atheroscler Thromb 2020.

36. Chiu TH, Huang YC, Chiu H, Wu PY, Chiou HC, Huang JC, Chen SC. Comparison of Various ObesityRelated Indices for Identification of Metabolic Syndrome: A Population-Based Study from Taiwan Biobank. Diagnostics (Basel) 2020, 10. 
37. Shin KA, Kim YJ. Usefulness Of Surrogate Markers Of Body Fat Distribution For Predicting Metabolic Syndrome In Middle-Aged And Older Korean Populations. Diabetes Metab Syndr Obes.

2019;12:2251-9.

\section{Figures}

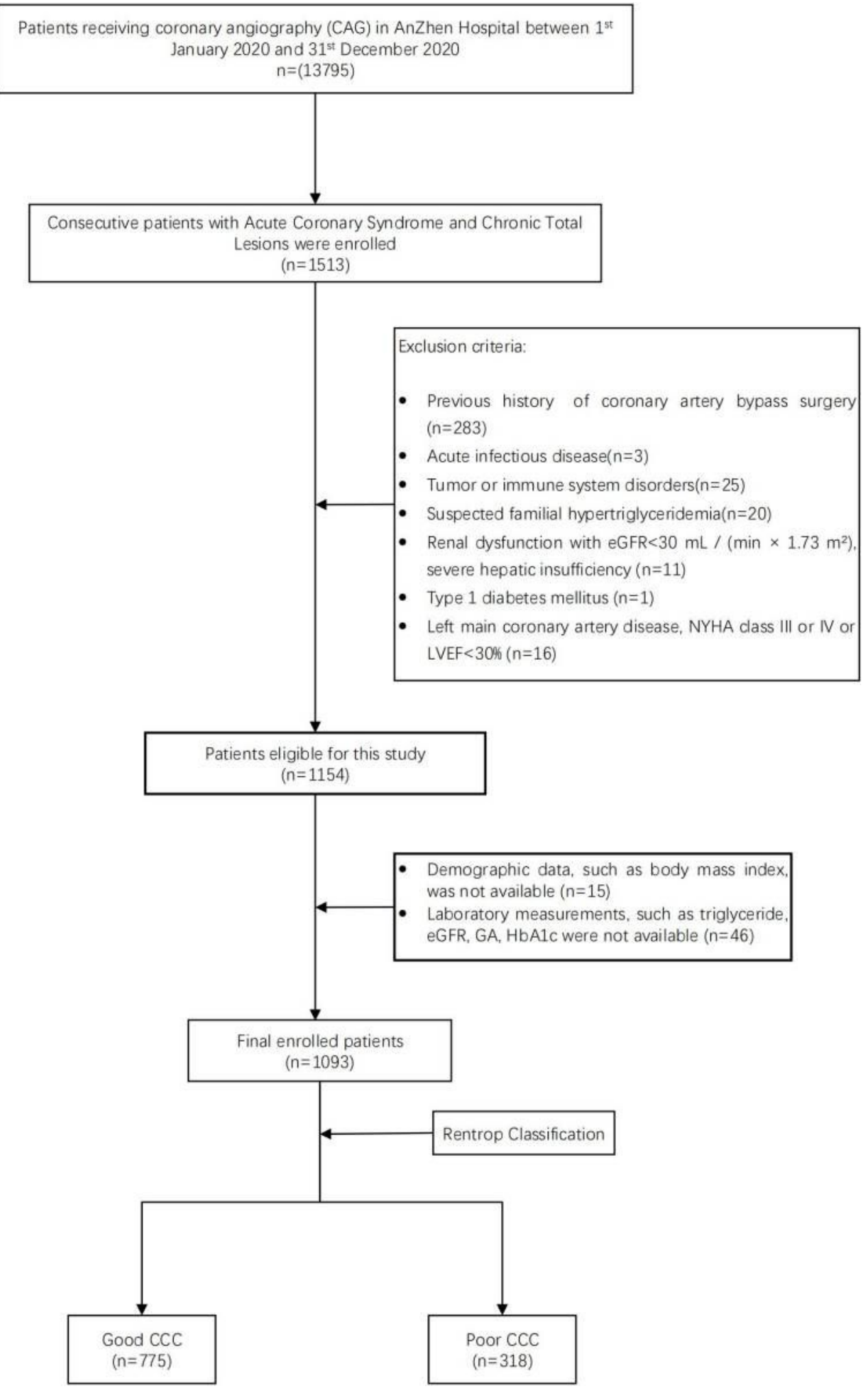

Figure 1

Page 21/25 
A total of 1513 patients who were diagnosed as CAD and with CTO (>3 months) of at least one major epicardial coronary artery at Beijing AnZhen Hospital between 1st Jan 2020 and 31st Dec 2020 were recruited into this single-center, observational study

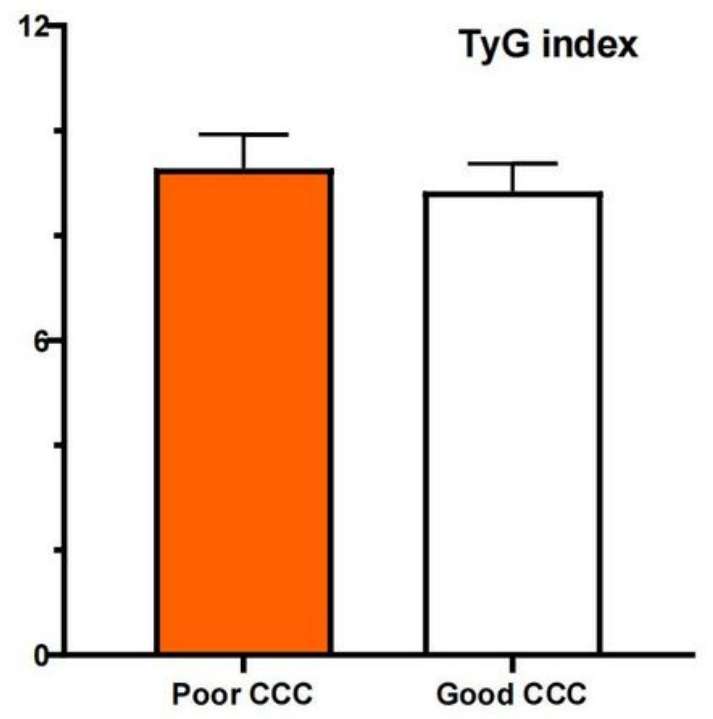

Figure 2A

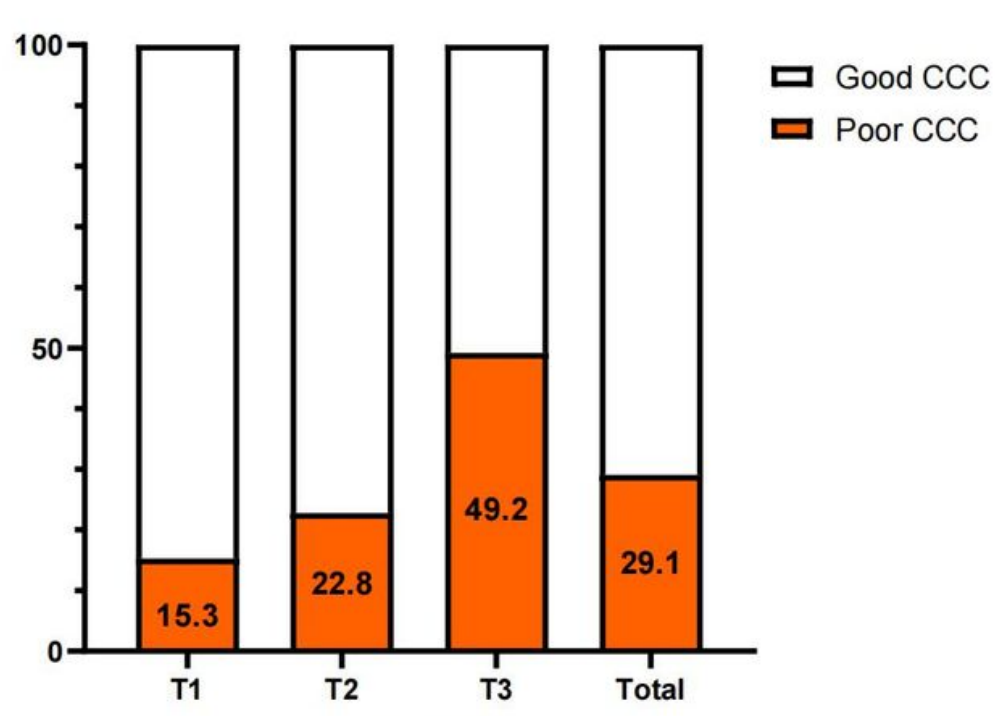

Figure 2B

\section{Figure 2}

TyG index in patients with poor collateralization was significantly higher than those with good one $(9.3 \pm 0.65$ vs. $8.8 \pm 0.53, P<0.001)$. Once dividing these participants into 3 tertiles according to the TyG index, the proportion of poor collateralization increased stepwise from the lowest TyG index tertile to the highest one ( $15.3 \%$ vs. $22.8 \%$ vs. $49.2 \%, P<0.001)$ 


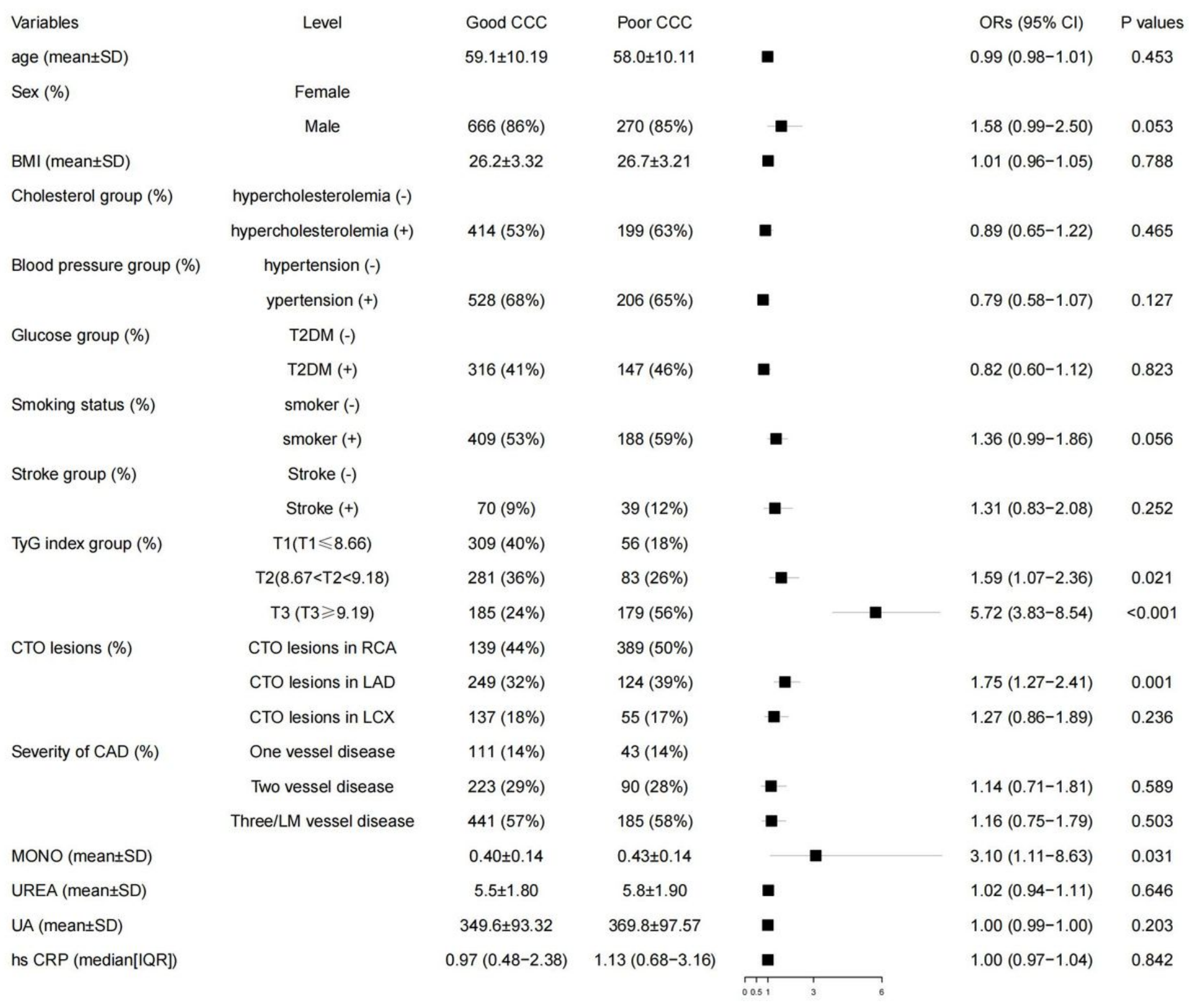

\section{Figure 3}

Three models were constructed to perform adjusted logistic regression analysis: Model1, after adjustment for age, sex, BMI, smoking history, Stroke, hypertension, T2DM and hypercholesterolemia; Model2, after adjustment for Model1 plus MONO, UREA, hs-CRP and LVEF; Model3, after adjustment for Model2 plus number of diseased vessels, CTO-related artery and ISR-CTO; The values of ORs ( $95 \% \mathrm{Cls}$ ) of T2 and T3 groups versus T1 were shown in Table3. The factors influencing the formation of CCC were shown in 


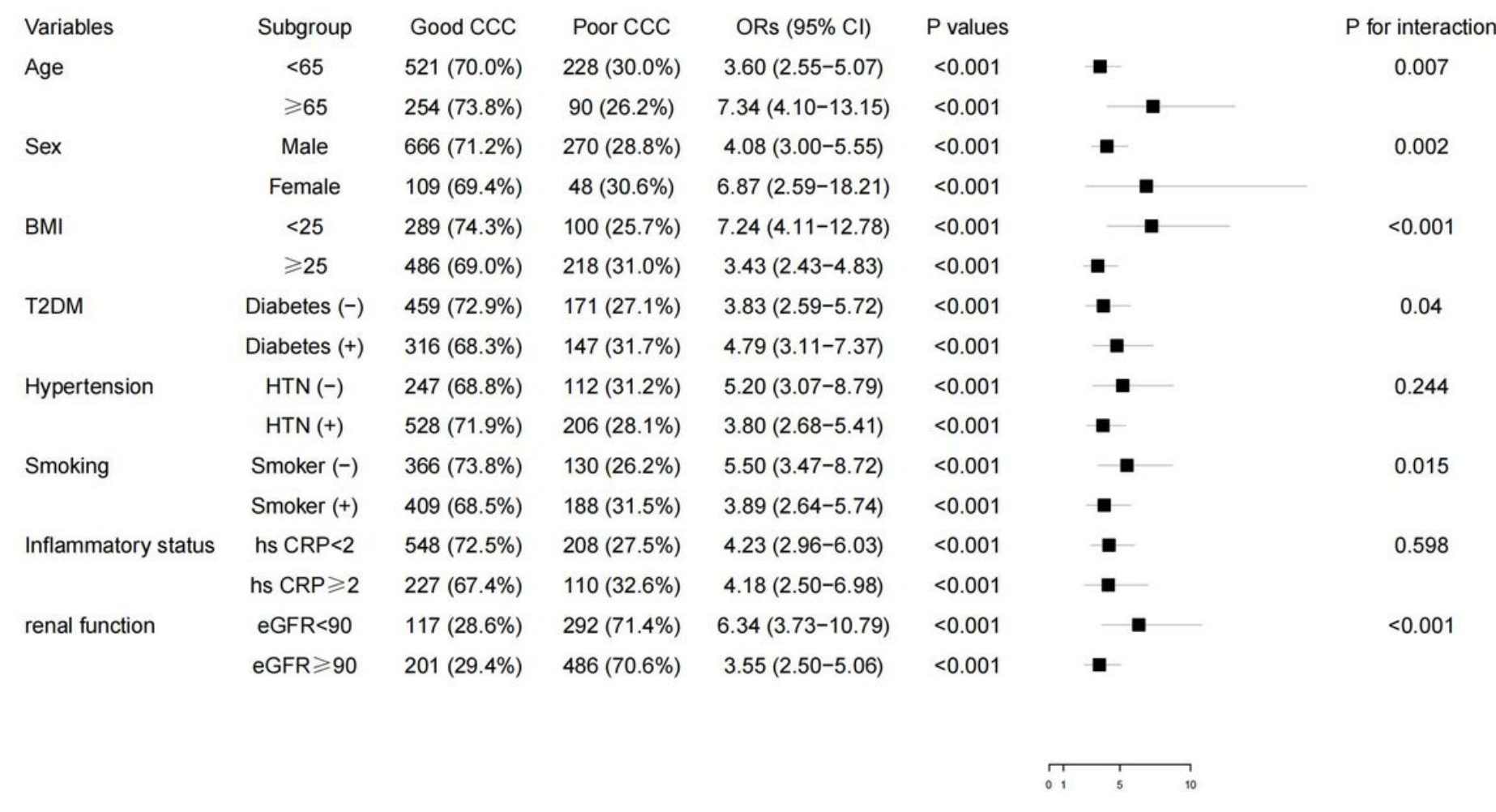

\section{Figure 4}

presented the results of subgroup analysis showing the relationship between TyG index and CCC in populations with different clinical characteristics. The adjusted ORs of TyG index for evaluating CCC remained significant across all subgroups. Finally, Additional file 2 showed the incremental diagnostic value of TyG index on the baseline model consisting of recognized risk factors related to poor CCC and its performance are better than $\mathrm{HbA} 1 \mathrm{c}$ and TG/HDL-C. The improvement of AUC for assessing the poor CCC was most significant when adding TyG index to the baseline model with a best
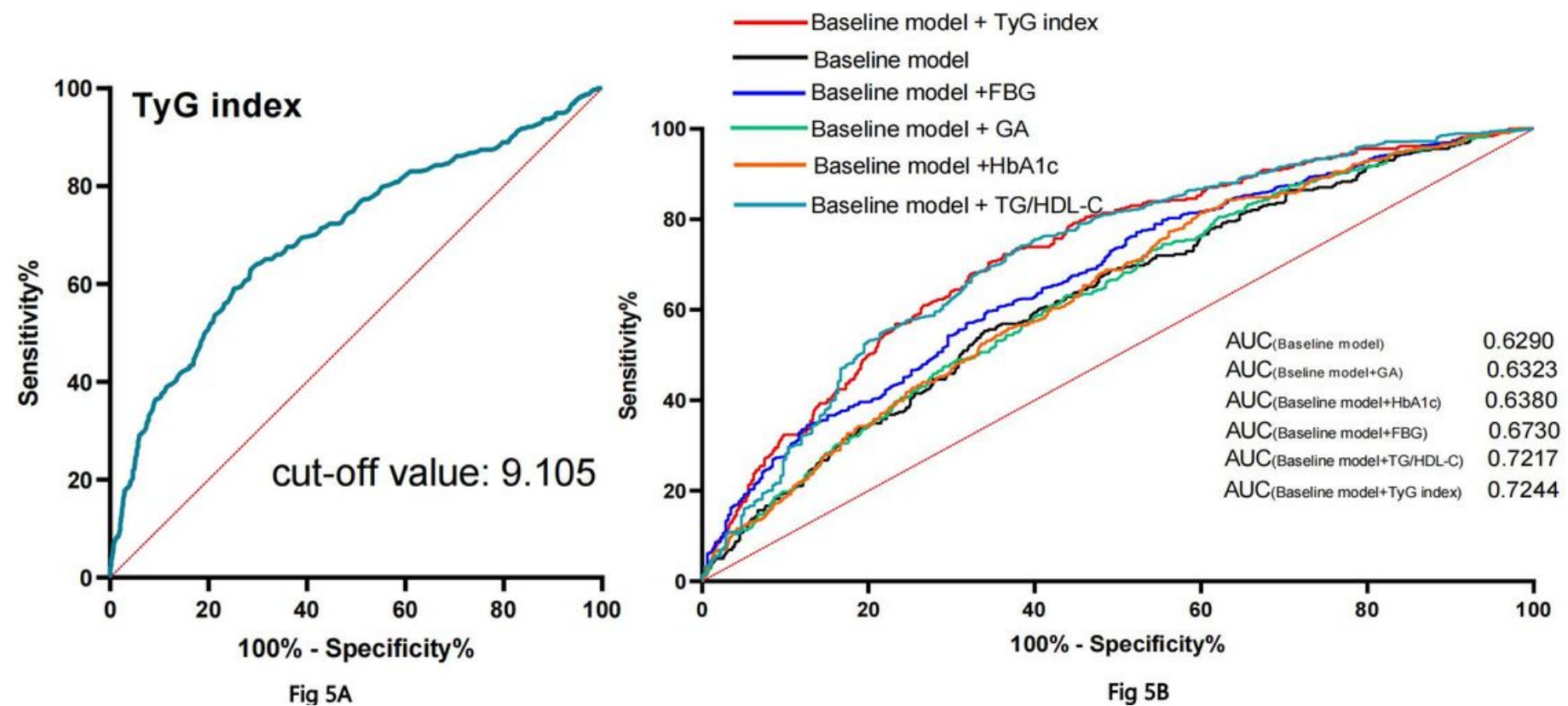

Fig 5B

Figure 5 
(AUC: baseline model 0.629 vs. +TyG index 0.724 ; baseline model 0.629 vs. +FPG 0.673 ; baseline model 0.629 vs. +GA 0.632; baseline model 0.629 vs. +HbA1c 0.638 ; baseline model 0.629 vs. +TG/HDL-C 0.721). Furthermore, the most significant enhancements on risk reclassification and discrimination were found after inclusion the TyG index into baseline model, with a NRI of $0.238(P<0.001)$ and an IDI of 0.103 $(P<0.001)$, when compared with FPG (NRI, 0.067, $P=0.009$; IDI, 0.037, $P<0.001)$ and TG/HDL-C (NRI, 0.159, $\mathrm{P}<0.001 ; \mathrm{IDI}, 0.087, \mathrm{P}<0.001)$

\section{Supplementary Files}

This is a list of supplementary files associated with this preprint. Click to download.

- Additionalfile1.docx

- Additionalfile2.docx 